\title{
Charge-Carrier Cooling and Polarization Memory Loss in Formamidinium Tin Triiodide
}

\author{
Kimberley J. Savill, ${ }^{\dagger}$ Matthew T. Klug, ${ }^{\dagger \odot}$ Rebecca L. Milot, ${ }^{\ddagger}$ Henry J. Snaith, ${ }^{\dagger}$ and Laura M. Herz ${ }^{*}{ }^{\dagger}(1)$ \\ ${ }^{\dagger}$ Department of Physics, University of Oxford, Clarendon Laboratory, Parks Road, Oxford OX1 3PU, U.K. \\ ${ }^{\ddagger}$ Department of Physics, University of Warwick, Gibbet Hill Road, Coventry CV4 7AL, U.K.
}

\section{Supporting Information}

ABSTRACT: Reports of slow charge-carrier cooling in hybrid metal halide perovskites have prompted hopes of achieving higher photovoltaic cell voltages through hot-carrier extraction. However, observations of long-lived hot charge carriers even at low photoexcitation densities and an orders-of-magnitude spread in reported cooling times have been challenging to explain. Here we present ultrafast time-resolved photoluminescence measurements on formamidinum tin triiodide, showing fast initial cooling over tens of picoseconds and demonstrating that a perceived secondary regime of slower cooling instead derives from electronic relaxation, state-filling, and recombination in the presence of energetic disorder. We identify limitations of some widely used approaches to determine charge-carrier temperature and make use of an improved model which accounts for the full photoluminescence line shape. Further, we do not find any persistent polarization anisotropy in $\mathrm{FASnI}_{3}$ within $270 \mathrm{fs}$ after excitation, indicating that excited carriers rapidly lose both polarization memory and excess energy through interactions

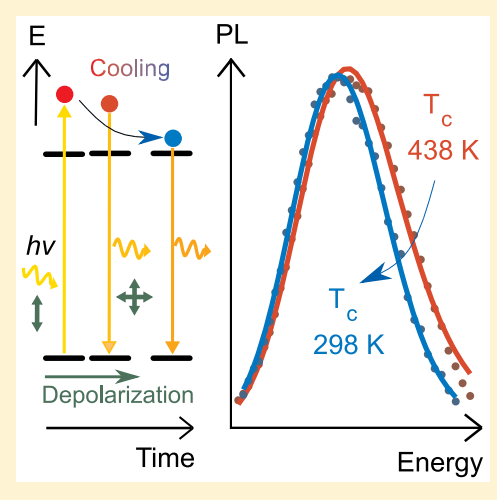
with the perovskite lattice.

Tn the last 10 years, solar cells using hybrid metal halide 1 perovskites $\left(\mathrm{ABX}_{3}\right)$ for light absorption have shown continuing increases in power conversion efficiency (PCE). Recently, lead-based $(\mathrm{B}=\mathrm{Pb})$ perovskite thin-film cells with efficiencies above $20 \%$ have been reported. ${ }^{1,2}$ Lead-free perovskite solar cells with tin as the metal cation also continue to improve and have recently achieved a certified PCE of $8.3 \%$ with a record of $9.6 \% .^{3}$ The stability of tin perovskite $(B=S n)$ solar cells has typically been a concern because of ready oxidation of $\mathrm{Sn}^{2+}$, which leads to substantial p-type doping ${ }^{4-6}$ and short charge-carrier lifetimes. ${ }^{6-10}$ Along with increased efficiencies, some improvements in stability have recently been reported through passivation by a native oxide layer, ${ }^{11}$ incorporation of a larger organic cation, ${ }^{3}$ or combining threedimensional (3D) with lower dimensionality perovskites in a single film. ${ }^{12}$ While tin perovskites still lag behind their leadbased counterparts in performance and are less well-studied from a fundamental perspective, these developments are important for potential future applications.

In particular, tin-containing perovskites are likely to play a role in the realization of device architectures beyond singlejunction cells. Tandem architecture devices, which combine absorbers with different bandgaps in order to reduce carriercarrier thermalization losses, are a promising example. Allperovskite tandem cells have achieved efficiencies exceeding $20 \%{ }^{13-15}$ for two-terminal tandems, and for four-terminal tandems up to $25 \%,{ }^{15}$ with the choice of low-bandgap absorber being an important contributor to performance improvements. Tin-lead perovskites are commonly used, as they exhibit bandgap bowing with even lower bandgaps for mixed
$\mathrm{APb}_{1-x} \mathrm{Sn}_{x} \mathrm{X}_{3}$ compositions than are seen with either lead or tin as the sole B cation. ${ }^{16}$ Several possible causes of this phenomenon have been suggested, ${ }^{13,17,18}$ and greater understanding of how the presence of tin affects the lattice structure and optoelectronic properties is likely to lead to further improvements in device performance in the future.

Another photovoltaic device concept offering the potential for higher power conversion efficiency is hot charge-carrier extraction, which theoretically offers up to 66\% PCE for a single-junction cell. ${ }^{19}$ Hot charge carriers are generated following above-bandgap absorption, which initially gives a nonequilibrium distribution of photoexcited charge carriers with excess kinetic energy. The excited charge carriers rapidly relax to the band edge, with carrier-carrier scattering resulting in an energetic distribution which can be characterized by a Boltzmann distribution with temperature $T_{c}$ that will initially be greater than the temperature of the lattice. This process of thermalization to a "hot" charge-carrier gas has been found to occur on a time scale of tens of femtoseconds for methlyammonium lead triiodide. ${ }^{20}$ The subsequent return of $T_{c}$ to the temperature of the lattice is referred to as chargecarrier cooling, and avoiding this energy loss pathway is key to the predicted increases in efficiency for a hot-carrier solar cell. ${ }^{19}$ Low-bandgap perovskites could see particularly large relative improvements in performance from hot charge-carrier extraction, as their absorption in the solar energy range

Received: August 12, 2019

Accepted: September 23, 2019

Published: September 23, 2019 
involves large excess energies, and so these materials are a natural choice for an investigation of charge-carrier cooling.

While hot charge-carrier extraction has not yet been realized in devices, the prospect of PCE improvement through this approach has motivated growing attention devoted to probing hot charge carriers in semiconducting perovskites. Reported time scales of charge-carrier cooling in metal halide perovskites range from subpicosecond $d^{21,22}$ to tens ${ }^{23,24}$ or hundreds ${ }^{25,26}$ of picoseconds up to nanoseconds. ${ }^{27}$ The longer cooling times reported are typically associated with a second stage of cooling that follows more rapid initial energy loss. ${ }^{27-29}$ In many cases slow cooling is attributed to a hot phonon bottleneck ${ }^{22,24,29-32}$ which may be in combination with Auger heating. ${ }^{28,33-35} \mathrm{Up}$ conversion of acoustic phonons to higher energy has been suggested to contribute to the formation of hot phonon bottlenecks. ${ }^{26}$ Other proposed origins of slow cooling include screening of hot charge carriers through large polaron formation, ${ }^{25}$ changes to density of states near the bandgap, ${ }^{27}$ and trapping of hot charge carriers. ${ }^{28}$ Local structural variations have also been raised as an important consideration for variations in cooling time. ${ }^{32}$ For a realistic assessment of the potential for hot-carrier photovoltaics with perovskite absorber layers and a full understanding of the lattice dynamics involved in cooling, it is critical that the reasons for the wide variation in reported cooling times are unravelled.

Here, we first examine critically some of the previously used approaches to the determination of charge-carrier temperatures from experimental data. We identify common approximations and evaluate their limitations when temperatures are calculated from experiment. We show that many of these issues can be overcome if an alternative model is used that accounts for the full photoluminescence (PL) line shape, including the correct density of electronic states, and broadening effects. Our improved method for carrier temperature determination hence addresses sources of error that may have contributed to the wide variation in reported cooling times for hybrid perovskites and offers a standardized approach to accurate fitting of PL spectra. Such an approach will ensure greater comparability between future studies, allowing for conclusive determination of charge-carrier cooling dynamics in promising perovskite materials and assessment of the potential for hot-carrier extraction in devices.

We further present an experimental study of charge-carrier cooling in formamidinium (FA) tin triiodide, $\mathrm{FASnI}_{3}$, prepared with the addition of $\mathrm{SnF}_{2}$ to moderate doping levels. Previous characterization of this perovskite has shown a low exciton binding energy of $3.1 \mathrm{meV}$; background hole density of $2.0 \times$ $10^{19} \mathrm{~cm}^{-3}$; and improvements in film quality, charge-carrier mobility, and lifetime attributable to $\mathrm{SnF}_{2}$ addition, giving a charge-carrier diffusion length of $165 \mathrm{~nm}$. Our ultrafast timeresolved photoluminescence measurements are conducted using PL upconversion spectroscopy with a setup previously described, giving 270 fs time resolution. ${ }^{36,37}$ To probe chargecarrier cooling in $\mathrm{FASnI}_{3}$ we determine temperatures by fits to the full PL spectra with our complete model accounting for energetic broadening and the correct near band-edge density of states. We find that following the rapid initial charge-carrier cooling over tens of picoseconds, an apparent slow decrease in temperature extending over hundreds of picoseconds is attributable to relaxation between energetically disordered states limited by overall carrier-carrier recombination dynamics, and not to exceptionally long-lived hot charge carriers. Finally, we examine polarization memory loss in
$\mathrm{FASnI}_{3}$ through measurements of the photoluminescence polarization anisotropy as a function of time after excitation. This technique offers an additional probe for the interactions of charge carriers with one another and with the structure and dynamics of the lattice. We observe zero PL polarization anisotropy already within the temporal resolution of our measurements. Our findings show that polarization memory in $\mathrm{FASnI}_{3}$ is rapidly lost on a time scale similar to thermalization, with charge-carrier cooling subsequently occurring over tens of picoseconds.

We begin our development of a robust approach to carrier temperature determination by examining the method which has been commonly used in previous studies and produced a wide range of values for cooling time in perovskites. In many recent studies, carrier temperature is determined by fitting of an exponential function $\left(\sim \exp \left(-E / k_{\mathrm{B}} T_{\mathrm{c}}\right)\right)$ to a selected "tail" region at the high-energy side of the measured emission peak. $^{20,22,24,30-35}$ This practice follows the approaches commonly used in previous work with inorganic semiconductors, such as GaAs or CdS, ${ }^{38-41}$ and their electronically confined quantum well systems in particular. ${ }^{42-51}$ Taking the Boltzmann distribution for thermalized charge carriers as a complete model for hot charge-carrier emission simplifies calculation by disregarding any other influences on the emission spectrum and would have been a particular advantage before the advent of computational power allowed more complex fits to data. However, today the available technology to analyze spectra is no longer a constraint, and as we show here, relying on the exponential-fit approach leads to several inaccuracies which present an obstacle to properly assessing the carrier cooling in hybrid perovskites.

There are two main sources of inaccuracies introduced by the simple method of exponential tail fits. First, we note that the commonly made assumption of a constant (energyindependent) electronic density of states is strictly valid only for the case of a quantum well (i.e., a two-dimensional density of states). ${ }^{42-51}$ For $3 \mathrm{D}$ bulk semiconductors this assumption does not apply, and so the distribution of charge carriers should be modeled with attention to the energy-dependent joint density of states for parabolic bands of a direct semiconductor $\left(J_{\mathrm{DOS}} \propto\left(E-E_{\mathrm{g}}\right)^{1 / 2}\right)$ as well as the simple thermal distribution. Using the approximation of a parabolic band structure near the band edge, which will hold for the relevant thermal energy range (see section S4.2 in the Supporting Information) gives a more suitable description for emission that includes such resulting energetic distribution of charge carriers in a bulk (3D) semiconductor. Second, measured PL spectra for metal halide perovskites display significant broadening with line widths greater than $100 \mathrm{meV}$ at room temperature in many cases. ${ }^{8,52-55}$ Causes of broadening include coupling of excited charge carriers with phonons, ${ }^{52,54,56,57}$ scattering with defects, ${ }^{8}$ and the presence of trap states. ${ }^{58,59}$ Spectral broadening affects not only the belowbandgap emission but also the line shape for the higher-energy side of the peak to which exponential tail fitting is commonly carried out. As we show below, if the correct joint density of states and the spectral broadening on line shape are not accounted for, extracted charge-carrier temperatures tend to be overestimated. In addition, we find that the exact region of the spectrum selected for such exponential tail fits introduces further variation in the temperatures determined.

We show here that determining charge-carrier temperature from a full-line shape model, with no further approximations 
made to the underlying emission profile previously identified, ${ }^{60,61}$ eliminates these weaknesses. The form of this model is given by eq 1 and is the convolution of a suitable function to describe the broadening of the measured spectrum, in this case a Gaussian distribution (see section S4.2 and Figure S5 in the Supporting Information), with the expression for spontaneous emission intensity $I_{\mathrm{PL}}$ given by eq 2 .

$$
\begin{aligned}
& I_{\text {measured }}(E)=\frac{1}{\sigma \sqrt{2 \pi}} \exp \left(\frac{-\left(E-E_{\mathrm{g}}\right)^{2}}{2 \sigma^{2}}\right) \otimes I_{\mathrm{PL}}(E) \\
& I_{\mathrm{PL}}(E) \propto \begin{cases}\left(E-E_{\mathrm{g}}\right)^{1 / 2} \exp \left[\frac{-\left(E-E_{\mathrm{g}}\right)}{k_{\mathrm{B}} T_{\mathrm{c}}}\right] & E>E_{\mathrm{g}} \\
0 & \text { otherwise }\end{cases}
\end{aligned}
$$

Together, eqs 1 and 2 define a full emission spectrum with intensity $I_{\text {measured }}$ at energy $E$. The parameters for the model are the bandgap of the emitting material, $E_{g}$; the broadening parameter $\sigma$ defining the width of the Gaussian distribution used; and the charge-carrier temperature, $T_{c}$. To demonstrate the impact on temperature determination of simplifying from this full line shape model to the commonly used exponential fit, we calculate emission spectra using eqs 1 and 2 for $T_{\mathrm{c}}$ from 5 to $1000 \mathrm{~K}$, with $E_{\mathrm{g}}$ and $\sigma$ values representative of our measured spectra. We also calculate spectra with an order of magnitude lower broadening than seen by experiment for the same range of temperatures. A high-energy tail region of each calculated spectrum is then used for simple exponential fitting to give a "tail fit temperature" for comparison to the known input temperature. From such comparison we are therefore able to analyze the systematic errors introduced by the tail-fit approximation.

Figure $1 \mathrm{~A}$ shows the calculated spectra at $T_{\mathrm{c}}=50,500$, and $1000 \mathrm{~K}$ according to eq 1 , for two values of $\sigma$ representing realistic $(\sigma=43 \mathrm{meV}$, fwhm $=102 \mathrm{meV})$ and low $(\sigma=5 \mathrm{meV}$, fwhm $=11.8 \mathrm{meV})$ broadening of the PL spectrum. Elevated charge-carrier temperatures contribute to both a blue shift in energy of the peak and asymmetry in the shape of the spectrum with shallower slope on the high-energy side of the peak than the low-energy side. With $\sigma=43 \mathrm{meV}$, the blue shift in peak position is greater, and the change in gradient at the highenergy side of the peak smaller than for $\sigma=5 \mathrm{meV}$ as $T_{\mathrm{c}}$ increases. It is visually apparent that a rise in charge-carrier temperature will result in starker relative changes in the PL line shape if the energetic broadening $\sigma$ is smaller. This is further illustrated in Figure S6A,B, which includes intermediate carrier temperatures across the range represented here.

Figure 1B illustrates the quality of fit that can be achieved when the selected tail region of each simulated spectrum is now fitted with either the exponential-tail approximation assuming a constant density of states $\left(I \propto \exp \left(-E / k_{\mathrm{B}} T_{\mathrm{c}}\right)\right)$ or the model given by eq 2 which includes an energy-dependent density of states but still ignores line shape broadening. The tail regions used for fitting and shown by + or $\times$ markers here are those marked in gray in Figure $1 \mathrm{~A}$, capturing points between $50 \%$ and $5 \%$ of peak intensity for each spectrum. The fitted curves diverge from the calculated spectra as energy increases, with more pronounced deviation between data and fits for lower charge-carrier temperature and for larger $\sigma$ values. The difference between the fits using the exponential-tail approximation (blue-black lines) and using eq 2 (light blue
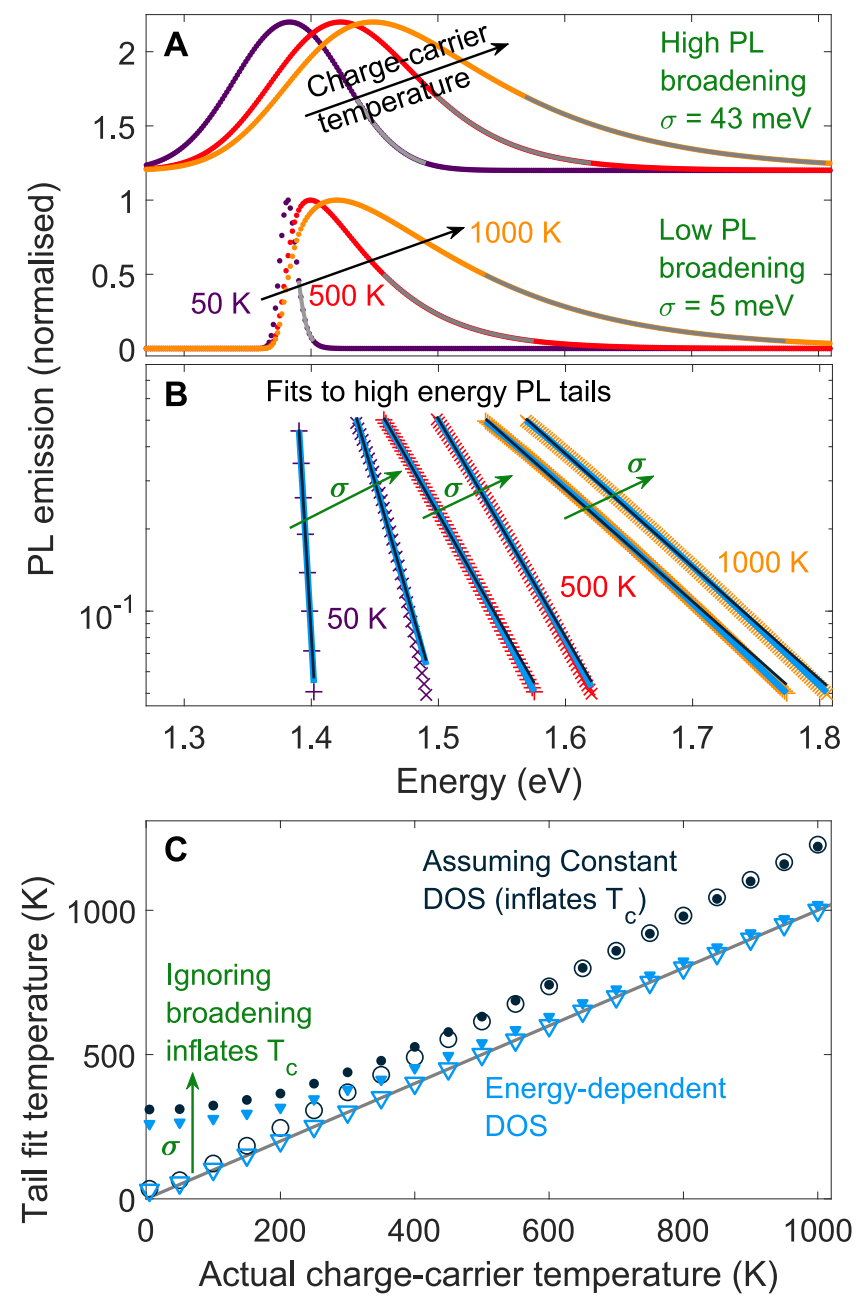

Figure 1. Comparison of models for carrier temperature. (A) Example photoluminescence spectra modeled using eq 1 for temperatures 50, 500 , and $1000 \mathrm{~K}$ with $E_{\mathrm{g}}=1.38 \mathrm{eV}$ and variable broadening parameter $\sigma$ valued $43.4 \mathrm{meV}$ (top set of spectra, vertical offset added) and 5 $\mathrm{meV}$ (bottom set of spectra, no vertical offset). Note that the parameter $\sigma$ relates to the full width at half-maximum (fwhm) of the peak through fwhm $=\sqrt{8 \ln 2} \sigma$. The same color scheme is used for both sets of spectra, denoting temperature as labeled on the figure. Tail regions selected to fit to, extending from $50 \%$ to $5 \%$ of the peak PL intensity, are shown by gray solid lines. (B) Selected tail regions and fits for the spectra as shown in panel A. Data points are plotted with + markers for $\sigma=5 \mathrm{meV}$ and with $\times$ markers for $\sigma=43.4 \mathrm{meV}$. Boltzmann model fits to each tail region are shown by thin blue-black lines. Fits using eq 2 to account for parabolic band structure, but not for broadening, are shown by thicker light blue lines. (C) Carrier temperatures from fits to spectra modeled as in panel A across a full range of input temperatures. Results for $\sigma=43.4 \mathrm{meV}$ are shown by point and filled triangle markers, and results for $\sigma=5 \mathrm{meV}$ are shown by $\bigcirc$ and $\nabla$ markers. The model used for fitting the tail is denoted by marker color, blue-black for the simple Boltzmann model with constant density of states and light blue for eq 2 with energydependent density of states, matching the fitted line colors in panel B. The gray solid line is a guide to the eye for equality between actual temperature used to produce the spectra and tail fit temperature.

lines) becomes more pronounced as temperature increases, showing closer agreement with the data when eq 2 is used. This, together with the differences in fitted temperatures shown in Figure 1C, illustrates the impact of correctly 
accounting for the $3 \mathrm{D}$ density of states in a semiconductor with parabolic bands.

To allow for a quantitative assessment of the systematic errors made under various scenarios, Figure 1C displays the charge-carrier temperatures extracted from exponential tail fits with the actual values entering as $T_{c}$ in the simulated spectra according to eq 1 . Here, the gray solid line indicates equality between the two values; it can therefore be seen immediately that the approximations discussed above result in the extraction of artificially elevated charge-carrier temperatures, with broadening primarily affecting low-temperature results and treatment of the density of states affecting hightemperature results. More detailed information can be gathered from inspection of the blue-black symbols which show the carrier temperatures extracted from typical exponential tail fits (i.e., assuming a constant density of states, and no spectral broadening) for the case of high (point markers) and low (open circles) actual broadening. Comparison of the two broadening scenarios shows that neglect of such spectral broadening through tail fitting leads to particularly erroneous results at low temperatures for realistically broadened spectra. The same trend is seen when comparing the temperatures fitted using eq 2 for the two broadening scenarios and plotted in light blue, with values significantly overestimated at low temperature when there is realistic broadening (filled triangle markers) and closer to the actual values for low broadening ( $\nabla$ markers). This finding is unsurprising, given that the relative magnitude of the quantity $k_{\mathrm{B}} T_{\mathrm{c}}$ with respect to the fwhm of the broadening influences the extent to which broadening can safely be ignored. We note that because most hybrid perovskite semiconductors exhibit fwhm of around $100 \mathrm{meV}$ at room temperature, such systematic overestimates of the actual charge-carrier temperatures when broadening is neglected are to be expected even for temperatures up to $1000 \mathrm{~K}$, which includes most of the scenarios reported in the literature.

In addition to the errors introduced by disregarding spectral broadening, we find that the neglect of the appropriate density of states becomes particularly detrimental at high chargecarrier temperatures. Figure $1 \mathrm{C}$ shows that if the joint density for parabolic bands $\left(J_{\mathrm{DOS}} \propto\left(E-E_{\mathrm{g}}\right)^{1 / 2}\right.$, as per eq 2$)$ for a bulk direct-gap semiconductors is correctly included, tail fits to spectra exhibiting low broadening (see light blue $\nabla$ markers) reproduce the actual charge-carrier temperatures fairly accurately. In the higher broadening case (light blue filled triangle markers), which is more reflective of spectra seen by experiment, tail fits accounting for the correct density of states also demonstrate good accuracy at high charge-carrier temperatures, but deviations at low temperature still persist because of the neglect of energetic broadening in excess of $k_{\mathrm{B}} T_{\mathrm{c}}$. This is further discussed in section S4.4 of the Supporting Information. In summary, the extraction of charge-carrier temperatures from simple exponential fitting to high-energy emission tails will lead to systematic overestimation of temperatures in the case of bulk hybrid perovskite semiconductors. At the low-temperature end, the neglect of spectral broadening dominates errors, while at the high-energy end the incorrect assumption of an energy-independent density of states leads to artificially elevated temperatures.

Aside from these two systematic errors discussed above, we find that the exact choice of the high-energy region of the emission tail to which exponential fits are applied can have a significant impact on the extracted charge-carrier temperatures (see section S4.3 of the Supporting Information for full discussion and figures). Exponential fits will tend to diverge at the higher-energy end of the chosen range where the absolute difference between fit and data is smaller because the signal has decayed, as can be seen in Figure 1B here and Figure S6 in the Supporting Information. In addition, because the gradient of any spectral line shape will flatten toward its peak, fits nearer the peak will return low gradients and therefore artificially elevated temperatures. Consequently, the closer the start of the selected range is to the PL peak, and the smaller the range used, the higher the extracted temperatures (see Figures S7 and $S 8$ for an illustration of this). Different methods of selecting the high-energy tail region for a set of time-resolved spectra, for example using a fixed energy range, ${ }^{22,24,29,35}$ ratios to the peak intensity, ${ }^{28}$ or an average over several selections, ${ }^{26}$ therefore introduce further variability in carrier temperatures and cooling dynamics determined from experiment. In transient absorption spectra, the ground-state bleach used to fit temperatures often overlaps with photoinduced absorption (PIA) features of opposite sign. A separate term for PIA is sometimes added to the exponential model when fitting longer tail ranges, ${ }^{62}$ while the overlap further complicates the choice of tail region for this specific technique.

The overestimates of carrier temperature shown in Figure 1 (and further in Figures S6 and S9) are not constant across the temperature range typically examined by experiment. The relative error in exponentially fitted carrier temperatures for realistically broadened spectra ranges from $22 \%$ at $1000 \mathrm{~K}$ to $46 \%$ at $300 \mathrm{~K}$, for example (see Figure S10). In combination with the dependence on the energy range that is fitted over this can lead to substantial variability and inaccuracy in temperatures extracted from simple exponential tail fits and hence erroneous reports of cooling dynamics. Fitting the full spectrum with eq 1 will eliminate these problems and provide an approach for carrier cooling studies that allow the true cooling dynamics of promising perovskite materials to be understood and meaningfully compared to one another. As a first step in this direction we now present our results for charge-carrier cooling dynamics in $\mathrm{FASnI}_{3}$.

Through a combination of ultrafast PL upconversion spectroscopy and time-correlated single-photon-counting measurements we are able to follow photoluminescence spectra of our $\mathrm{FASnI}_{3}$ films from subpicosecond out to nanosecond time scales. Figure $2 \mathrm{~A}$ shows a representative selection of spectra over a range of delay times, in which the typical hot-carrier PL profile can be seen. The high-energy side of the peak is initially elevated in intensity, dropping away to give a more symmetrical profile at later times as charge carriers cool (Figure 2A). To extract carrier temperatures we fit the measured spectra at each time after excitation using eq 1 with fixed values of $E_{\mathrm{g}}$ and $\sigma$ determined from global fits to fully cooled spectra for each excitation fluence, and the parameter of interest $T_{\mathrm{c}}$ is free to vary for each spectrum (see section S4.5 of the Supporting Information for full details of the procedure and more examples of measured and fitted spectra). The carrier temperatures determined in this way are plotted in dark blue as cooling curves in Figure 2B,C, for two different excitation fluences. The initial temperatures from our measurements are around $450 \mathrm{~K}$, which is somewhat colder than in several previous reports for similar initial carrier densities across a range of metal halide compositions, but on the same order of magnitude in many cases, ${ }^{22,24,26,34,62}$ with only a few reports of temperatures exceeding $1000 \mathrm{~K}^{28,30,35}$ Lower 

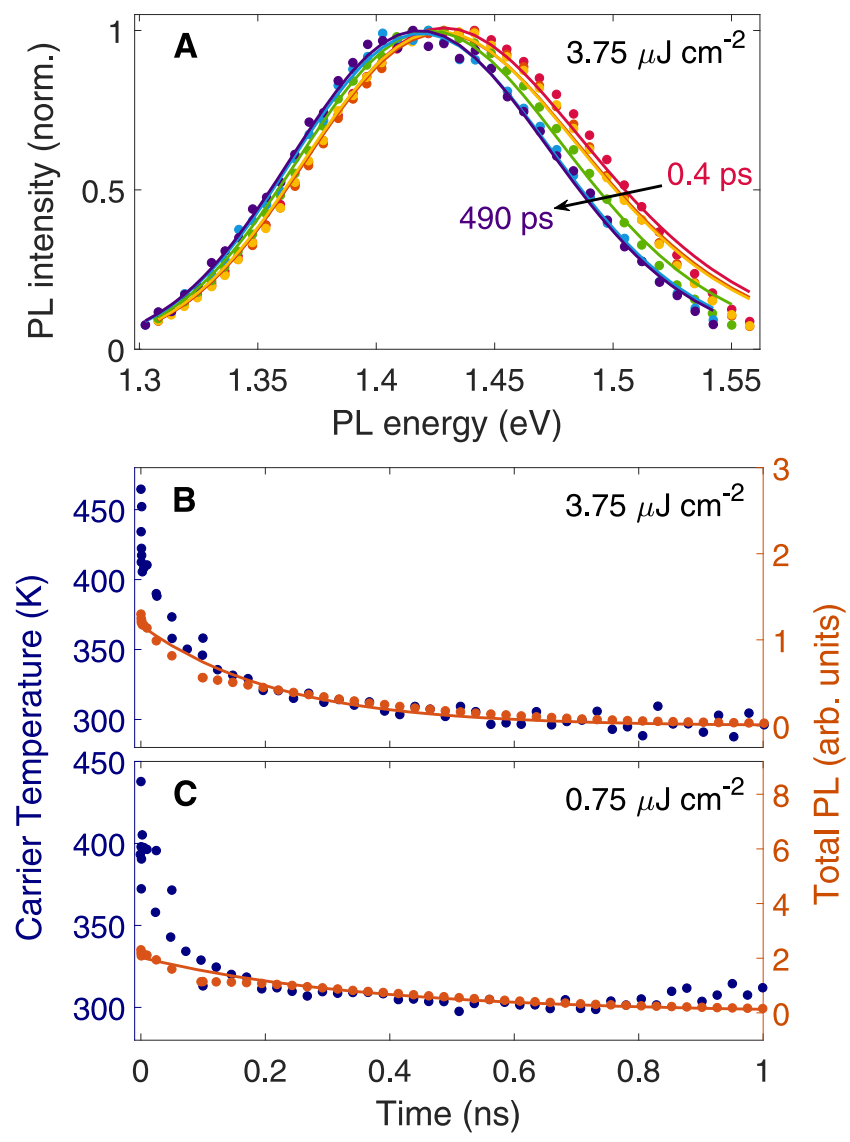

Figure 2. Charge-carrier cooling and PL decay dynamics of $\mathrm{FASnI}_{3}$ films prepared with $5 \% \mathrm{SnF}_{2}$, taken under pulsed laser excitation at $730 \mathrm{~nm}$ with fluences as marked. (A) Representative spectra for 3.75 $\mu \mathrm{J} \mathrm{cm} \mathrm{cm}^{-2}$ excitation as used to determine carrier temperatures plotted in panel B. Point markers show the measured data, and solid lines show the fits using the full line shape model given by eqs 1 and 2. (B and C) Cooling curves (blue point markers) overlaid with total PL decay curves (orange point markers), for the high- and low-fluence conditions, respectively. Carrier temperatures are given by fits as shown in panel $\mathrm{A}$, following the process described in full in section S4.5 of the Supporting Information. Total PL data are calculated by integrating over the full energy range for each measured spectrum. Monoexponential fits to the total PL decay transients are shown by orange solid lines.

temperatures are to be expected from our full spectrum fit in comparison to the exponential tail fit approach which yields the systematic overestimation already described, and some cooling may also have already occurred within the $270 \mathrm{fs}$ time resolution of our measurements.

Examining the dynamics shown in Figure 2B,C reveals that for both excitation fluences charge carriers rapidly cool over tens of picoseconds, and the two cooling curves are similar. Charge-carrier temperatures for the higher excitation fluence of $3.75 \mu \mathrm{J} \mathrm{cm}^{-2}$ are slightly higher than for the low fluence until around $0.4 \mathrm{~ns}$, and in both cases temperatures above $300 \mathrm{~K}$ persist to later times. The initial charge-carrier densities averaged across the $\mathrm{FASnI}_{3}$ film are $6.9 \times 10^{16}$ and $3.4 \times 10^{17}$ $\mathrm{cm}^{-3}$, respectively, for our two excitation fluences (see section S1.4 of the Supporting Information for details of this calculation). These values are below the thresholds reported elsewhere for hot phonon bottleneck effects, ${ }^{22,24,28,30}$ and so rapid cooling such as we observe initially would be expected if in fact only the phonon bottleneck could contribute to slowing cooling. Following the initial drop, our extracted carrier temperatures take several hundreds of picoseconds to level out at $300 \mathrm{~K}$ as a fully cooled carrier population. Previous reports of similar slow cooling without a buildup of hot phonons have suggested that influences such as lattice screening or Rashbalike effects result in unusually long-lived hot carriers. ${ }^{25,27}$ However, a different explanation is suggested if one compares the dynamics of this apparent residual cooling against the overall decay of the charge-carrier population as reflected in the associated photoluminescence. The data points shown in orange in Figure 2B,C are total PL intensities, calculated by integration across the full measured spectral range and capturing the decay of the full charge-carrier population over time. As can be seen from the figure, the early cooling dynamics are distinct from this overall decay, while the slower change over hundreds of picoseconds closely tracks the decay in total PL intensity.

This comparison implies that the changes to the spectrum at late time which yield still slightly elevated carrier temperatures, namely a reduction in intensity at the high-energy side of the peak and some apparent redshift, are connected to the overall decrease in charge-carrier population in a way that the rapid early cooling is not. We identify a mechanism for this observation, in the form of slow relaxation through energetically disordered states which is limited by charge-carrier occupation in the lower-energy band tail states. Such a process, occurring independently from the thermalization and subsequent cooling of free, delocalized charge carriers, explains the observed results and is consistent with known properties of tin halide perovskites. Specifically, the tendency of $\mathrm{Sn}^{2+}$ to oxidize to $\mathrm{Sn}^{4+}$ and introduce background hole doping in tin perovskites ${ }^{7,63}$ will lead to local variations in the electrostatic environment. The addition of $\mathrm{SnF}_{2}$ during fabrication of our samples reduces the dopant density, ${ }^{6}$ but it has yet to be established how this additive is distributed across the perovskite film; ${ }^{64}$ inhomogeneities are likely to introduce further local energetic variation across our samples. Additionally, random orientations of organic cations throughout the film contribute to lattice distortions and electrostatic variation ${ }^{65-68}$ with greater distortion around FA cations than the smaller methylammonium (MA) cation. ${ }^{69}$ As a consequence of disorder, states with energies distributed both above and below the bandgap are introduced to the energy landscape of the film, contributing to a band tail of below gap states ${ }^{70,71}$ as well as a distribution of states at higher energy which may be occupied by carriers with excess energy after photoexcitation. The existence of such a distribution is supported by comparison of PL decay dynamics across the spectrum (see Figure S17) which show slower decay at lower energies across the spectrum, consistent with relaxation of carriers between excited states over time preserving a larger population in lowenergy states. Such state filling at lower energies results in relaxation between disordered energetic states that is limited by decay of the charge-carrier population through recombination. We note that such a mechanism will even be operational at the low charge-carrier densities employed here, for which simple band-filling effects are likely to be minor. ${ }^{72-74}$ The observed longer-lived high-energy PL and the slow decline in fitted temperatures matching overall PL decay dynamics are in strong support of this argument.

We conclude, therefore, that the hot-carrier population in $\mathrm{FASnI}_{3}$ generated by moderately above-bandgap excitation actually cools over a tens of picoseconds time scale and that a 

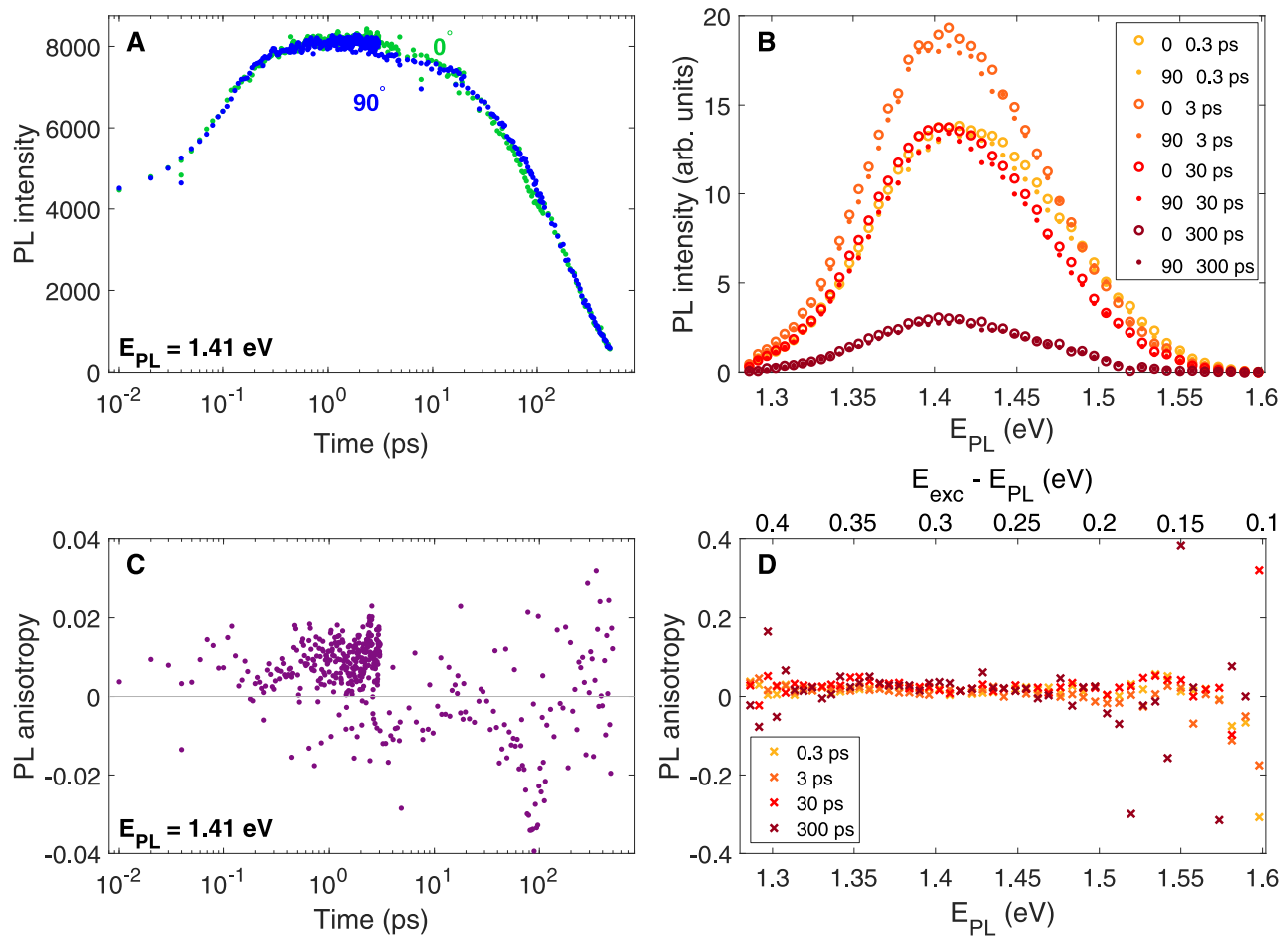

Figure 3. Time evolution and energy dependence of polarization anisotropy in the photoluminescence from a FASnI ${ }_{3}$ thin film following excitation at $730 \mathrm{~nm}$ with $0.75 \mu \mathrm{J} \mathrm{cm}^{-2}$ excitation. (A) Photoluminescence decay profiles for emission at $1.41 \mathrm{eV}$. Data for $0^{\circ}$ polarization (the condition of PL polarization parallel to the excitation polarization) are shown in green, and for $90^{\circ}$ (the perpendicular condition) in blue. (B) Spectra measured at delay times from 0.3 to $300 \mathrm{ps}$ after excitation, with the $90^{\circ}$ data (point markers) scaled against $0^{\circ}$ data (open circles) at the peak to match the calculated ratio $I_{\perp} / I_{\|}$. (C) Calculated polarization anisotropies across all delay data for emission at $1.41 \mathrm{eV}$ (purple point markers) with zero anisotropy line included (light gray, solid) as a guide to the eye. (D) Calculated polarization anisotropies across the spectral range for each time delay, illustrating the lack of dependence on emission energy and therefore absent difference between excitation and emission energies. The marker color scheme used here matches that in panel B.

separate process of relaxation through localized energetic states arising from disorder is responsible for the erroneous appearance of slow cooling at later times. In general, such population decay in an energetically disordered semiconductor, compounded by simplifications in the approach to temperature fitting, therefore has the potential to yield artificially elevated carrier temperatures even at excitation densities for which a phonon bottleneck should not be expected to occur.

Through further time-resolved PL measurements with polarization selectivity both parallel and perpendicular to the excitation beam polarization, we also investigate the $\mathrm{PL}$ polarization memory loss for $\mathrm{FASnI}_{3}$. Linearly polarized light excites transitions with a transition dipole matrix element in the direction of the polarization, which may result in an anisotropic distribution of charge carriers with correspondingly polarized emission. This initial polarization anisotropy may be lost over time, with the rate of memory loss depending on the nature of the initial selectivity. For materials with transition dipoles aligned to molecular structure, polarization memory loss can result from molecular reorientation, ${ }^{75}$ structural distortion, ${ }^{37}$ energy transfer to another emissive molecule in a different orientation, ${ }^{76}$ delocalization following excitation, ${ }^{37}$ or migration of charges along and between molecular subunits. ${ }^{77}$ In a bulk semiconductor, if there are no structural anisotropies leading to polarization-selective absorption, then the initial anisotropy in excited charge-carrier momenta can be lost in tens of femtoseconds through carrier-carrier scattering as is seen in GaAs. ${ }^{78,79}$ Tracking the loss of polarization memory is therefore a useful probe for the existence of any structural features coupling to polarization and the evolution of such features over time. Some lead halide perovskites have recently been found to display polarization anisotropy in transient absorption measurements lasting several picoseconds and attributed to local lattice dynamics with strong coupling to optical properties. ${ }^{80}$ As we have already discussed, local variations in the lattice of $\mathrm{FASnI}_{3}$ are to be expected, and these contribute to energetic disorder, so it is interesting to investigate whether there are also impacts on polarization memory from such factors and if they are dynamic. More generally, perovskite lattice distortions are important to other properties, including to carrier-cooling where the phonons to which energy is transferred are key.

Results of our measurements for PL polarization anisotropy, following the procedure described in section S6 of the Supporting Information, are shown in Figure 3. We examine emission intensity with respect to both delay time (panels A and C) and energy (panels B and D). The PL polarization anisotropy $r$ is calculated from photoluminescence intensity polarized either parallel $\left(I_{\|}\right)$or perpendicular $\left(I_{\perp}\right)$ to the polarization of the excitation beam according to eq 3 :

$$
r=\frac{I_{\|}-I_{\perp}}{I_{\|}+2 I_{\perp}}
$$

As shown in Figure 3C,D, the calculated PL anisotropy has values close to zero from very early times and remains close to zero across the several hundred picosecond time range covered by our measurements. For all but a few stray points the calculated anisotropy also remains within experimental 
uncertainty (0.044, see Figure S19) of zero, and we conclude that there is no polarization memory retained beyond the 270 fs resolution of our system, nor any evolution over the time scales of carrier cooling or PL decay. Similarly, measured spectra at each delay time investigated (Figure 3B,D) show zero anisotropy across the full energy range. We note that for later measurements the low signal above $1.5 \mathrm{eV}$ contributes to high noise in the calculated anisotropy values.

To contextualize these results, we first consider a recent study demonstrating polarization memory persisting for several picoseconds in methylammonium lead triiodide or tribromide and $\mathrm{CsPbBr}_{3} .{ }^{80}$ The proposed explanation for the observed PL polarization anisotropy in that work is the existence of locally polarized regions of the lead halide perovskite film, each with a defined Rashba direction and varying over time as the lattice distorts, so that the corresponding changes in local electronic structure which give polarization memory also change over time. ${ }^{80}$ Dynamics of the lattice involved in this local symmetry breaking and polarization in lead perovskites have been identified as involving distortions of the inorganic octahe$\mathrm{dra}^{81,82}$ which may be coupled to organic cation orientation. ${ }^{67,83,84}$ The spin-orbit coupling which gives rise to the Rashba effect at a local scale in these cases is stronger for lead than for the lighter tin cation in our $\mathrm{FASnI}_{3}$ samples, ${ }^{85}$ and on this basis some reduction in anisotropy relative to reports for $\mathrm{MAPbI}_{3}$ and related perovskites might be expected. Additionally, to the extent that cation motion drives lattice distortions, the impact of changing from methylammonium or cesium to formamidinium must be considered. In lead-based perovskites, FA reorientation has been experimentally determined to occur an order of magnitude faster than reorientation of $\mathrm{MA},{ }^{86}$ which would then correspond to shorter-lived local polarization and rapid loss of memory such as we observe. We note however that the converse, i.e., predictions of faster reorientation for MA than $\mathrm{FA}^{87}$ as well as experimental rates showing little difference between the two at room temperature ${ }^{88}$ have also been reported. The true impact of the change in cation is therefore uncertain, but the lack of polarization memory observed in our measurements does indicate that any local polarization coupling to electronic states is too short-lived to be detectable with a $270 \mathrm{fs}$ time resolution interval.

Symmetry of the perovskite lattice structure has previously been suggested to account for observations of polarization memory lasting hundreds of picoseconds in tetragonal methylammonium lead triiodide with no polarization memory in the cubic mixed iodide-chloride or iodide-bromide compositions. $^{89}$ We note that this does not explain the observation of shorter lived polarization memory in all three of $\mathrm{MAPbBr}_{3}, \mathrm{MAPbI}_{3}$, and $\mathrm{CsPbI}_{3}{ }^{80}$ given that $\mathrm{MAPbBr}_{3}$ is reported to be cubic at room temperature. ${ }^{90,91}$ Moreover, distinguishing cubic from pseudocubic structures can be challenging given the disorder in organic cation orientation and position. The structure of $\mathrm{FASnI}_{3}$ has been variously assigned as cubic ${ }^{92,93}$ or orthorhombic ${ }^{63,94,95}$ at room temperature, and as such we find that macroscale structural considerations do not offer a clear explanation for the observed absence of polarization anisotropy in our measurements. In the absence of structural contributions to polarization memory any initially anisotropic distribution of carrier momenta is expected to be rapidly lost through scattering of carriers, a process which has been reported on time scales down to tens of femtoseconds in GaAs, for example, ${ }^{78,79}$ and as such would fall within the 270 fs instrument response time of our measurements. Further work to characterize lattice dynamics in $\mathrm{FASnI}_{3}$ might answer the question of whether any existing local distortions in lattice structure change on too fast a time scale to impact observed polarization of photoluminescence or do not lead to polarization in electronic structure. From our results we can conclude that any local structural changes must fall under one or both of those cases and that in this respect the properties of $\mathrm{FASnI}_{3}$ differ from those of several lead halide perovskites.

In conclusion, we have investigated the dynamics of polarization memory loss and charge-carrier temperature in $\mathrm{FASnI}_{3}$ at moderate photoexcited carrier densities. We find that charge-carrier cooling is dominated by initial rapid relaxation over tens of picoseconds. The appearance of slower cooling at late times is not representative of a population of long-lived hot charge carriers and instead is attributable to relaxation between energetically disordered states dependent upon underlying recombination dynamics. Polarization memory is lost on a time scale much shorter $(<270 \mathrm{fs})$ than that for carrier cooling and does not vary with energy across the observed spectral emission range. These results suggest that scattering of charge carriers with other carriers and with phonons occurs freely and without screening. Further, local variations in the lattice have an impact on the energetic landscape which must be considered when interpreting chargecarrier cooling curves but do not result in any long-lived polarization anisotropy. In addition to developing an understanding of the properties of this lead-free perovskite, we have presented an improved model for determining carrier temperature addressing three key sources of error introduced by oversimplification. Spectral broadening is accounted for to avoid errors at low carrier temperatures; the assumption of a constant density of states which leads to increasing overestimation of temperatures for hotter distributions is replaced by an expression appropriate for direct transitions between parabolic bands, and the dependence on choice of spectral region to fit is eliminated. The adoption of this full line shape model offers a path to more accurate and readily comparable results in future studies of carrier cooling, so that the true potential for hot-carrier solar cells can be accurately assessed.

\section{ASSOCIATED CONTENT}

\section{S Supporting Information}

The Supporting Information is available free of charge on the ACS Publications website at DOI: 10.1021/acs.jpclett.9b02353.

Experimental details, film characterization (SEM images, thickness measurement, and XRD spectra), absorbance spectrum, further discussion of full-spectrum model for carrier temperature determination, details of fitting procedure, additional PL decay curves, and further details of polarization anisotropy measurements including error analysis (PDF)

\section{AUTHOR INFORMATION}

\section{Corresponding Author}

*E-mail: laura.herz@physics.ox.ac.uk.

ORCID

Matthew T. Klug: 0000-0002-9177-4313

Henry J. Snaith: 0000-0001-8511-790X

Laura M. Herz: 0000-0001-9621-334X 


\section{Notes}

The authors declare no competing financial interest.

\section{ACKNOWLEDGMENTS}

The authors acknowledge financial support from the Engineering and Physical Sciences Research Council (EPSRC), UK. K.J.S. thanks the Rhodes Trust for financial support through a Rhodes Scholarship. L.M.H. thanks the Humboldt Foundation for support through a Friedrich-Wilhelm-Bessel Research Award.

\section{REFERENCES}

(1) Green, M. A.; Hishikawa, Y.; Dunlop, E. D.; Levi, D. H.; HohlEbinger, J.; Yoshita, M.; Ho-Baillie, A. W. Y. Solar Cell Efficiency Tables (Version 53). Prog. Photovoltaics 2019, 27, 3-12.

(2) Jiang, Q.; Zhao, Y.; Zhang, X.; Yang, X.; Chen, Y.; Chu, Z.; Ye, Q.; Li, X.; Yin, Z.; You, J. Surface Passivation of Perovskite Film for Efficient Solar Cells. Nat. Photonics 2019, 13, 460-466.

(3) Jokar, E.; Chien, C.-H.; Tsai, C.-M.; Fathi, A.; Diau, E. W.-G. Robust Tin-Based Perovskite Solar Cells with Hybrid Organic Cations to Attain Efficiency Approaching 10\%. Adv. Mater. 2019, $31,1804835$.

(4) Konstantakou, M.; Stergiopoulos, T. A Critical Review on Tin Halide Perovskite Solar Cells. J. Mater. Chem. A 2017, 5, 1151811549 .

(5) Leijtens, T.; Prasanna, R.; Gold-Parker, A.; Toney, M. F.; McGehee, M. D. Mechanism of Tin Oxidation and Stabilization by Lead Substitution in Tin Halide Perovskites. ACS Energy Lett. 2017, 2, 2159-2165.

(6) Milot, R. L.; Klug, M. T.; Davies, C. L.; Wang, Z.; Kraus, H.; Snaith, H. J.; Johnston, M. B.; Herz, L. M. The Effects of Doping Density and Temperature on the Optoelectronic Properties of Formamidinium Tin Triiodide Thin Films. Adv. Mater. 2018, 30, 1804506.

(7) Noel, N. K.; Stranks, S. D.; Abate, A.; Wehrenfennig, C.; Guarnera, S.; Haghighirad, A.-A.; Sadhanala, A.; Eperon, G. E.; Pathak, S. K.; Johnston, M. B.; et al. Lead-Free Organic-Inorganic Tin Halide Perovskites for Photovoltaic Applications. Energy Environ. Sci. 2014, 7, 3061-3068.

(8) Parrott, E. S.; Milot, R. L.; Stergiopoulos, T.; Snaith, H. J.; Johnston, M. B.; Herz, L. M. Effect of Structural Phase Transition on Charge-Carrier Lifetimes and Defects in $\mathrm{CH}_{3} \mathrm{NH}_{3} \mathrm{SnI}_{3}$ Perovskite. J. Phys. Chem. Lett. 2016, 7, 1321-1326.

(9) Song, T.-B.; Yokoyama, T.; Stoumpos, C. C.; Logsdon, J.; Cao, D. H.; Wasielewski, M. R.; Aramaki, S.; Kanatzidis, M. G. Importance of Reducing Vapor Atmosphere in the Fabrication of Tin-Based Perovskite Solar Cells. J. Am. Chem. Soc. 2017, 139, 836-842.

(10) Handa, T.; Yamada, T.; Kubota, H.; Ise, S.; Miyamoto, Y.; Kanemitsu, Y. Photocarrier Recombination and Injection Dynamics in Long-Term Stable Lead-Free $\mathrm{CH}_{3} \mathrm{NH}_{3} \mathrm{SnI}_{3}$ Perovskite Thin Films and Solar Cells. J. Phys. Chem. C 2017, 121, 16158-16165.

(11) Chen, M.; Ju, M.-G.; Garces, H. F.; Carl, A. D.; Ono, L. K.; Hawash, Z.; Zhang, Y.; Shen, T.; Qi, Y.; Grimm, R. L.; et al. Highly Stable and Efficient All-Inorganic Lead-Free Perovskite Solar Cells with Native-Oxide Passivation. Nat. Commun. 2019, 10, 16.

(12) Wang, F.; Jiang, X.; Chen, H.; Shang, Y.; Liu, H.; Wei, J.; Zhou, W.; He, H.; Liu, W.; Ning, Z. 2D-Quasi-2D-3D Hierarchy Structure for Tin Perovskite Solar Cells with Enhanced Efficiency and Stability. Joule 2018, 2, 2732-2743.

(13) Eperon, G. E.; Leijtens, T.; Bush, K. A.; Prasanna, R.; Green, T.; Wang, J. T.-W.; McMeekin, D. P.; Volonakis, G.; Milot, R. L.; May, R; et al. Perovskite-Perovskite Tandem Photovoltaics with Optimized Band Gaps. Science 2016, 354, 861-865.

(14) Zhao, D.; Chen, C.; Wang, C.; Junda, M. M.; Song, Z.; Grice, C. R.; Yu, Y.; Li, C.; Subedi, B.; Podraza, N. J.; et al. Efficient TwoTerminal All-Perovskite Tandem Solar Cells Enabled by High-Quality Low-Bandgap Absorber Layers. Nat. Energy 2018, 3, 1093.
(15) Tong, J.; Song, Z.; Kim, D. H.; Chen, X.; Chen, C.; Palmstrom, A. F.; Ndione, P. F.; Reese, M. O.; Dunfield, S. P.; Reid, O. G.; et al. Carrier Lifetimes of $>1 \mu \mathrm{s}$ in $\mathrm{Sn}-\mathrm{Pb}$ Perovskites Enable Efficient AllPerovskite Tandem Solar Cells. Science 2019, 364, 475-479.

(16) Hao, F.; Stoumpos, C. C.; Chang, R. P. H.; Kanatzidis, M. G. Anomalous Band Gap Behavior in Mixed $\mathrm{Sn}$ and $\mathrm{Pb}$ Perovskites Enables Broadening of Absorption Spectrum in Solar Cells. J. Am. Chem. Soc. 2014, 136, 8094-8099.

(17) Im, J.; Stoumpos, C. C.; Jin, H.; Freeman, A. J.; Kanatzidis, M. G. Antagonism between Spin-Orbit Coupling and Steric Effects Causes Anomalous Band Gap Evolution in the Perovskite Photovoltaic Materials $\mathrm{CH}_{3} \mathrm{NH}_{3} \mathrm{Sn}_{1-x} \mathrm{~Pb}_{x} \mathrm{I}_{3}$. J. Phys. Chem. Lett. 2015, 6, 3503-3509.

(18) Parrott, E. S.; Green, T.; Milot, R. L.; Johnston, M. B.; Snaith, H. J.; Herz, L. M. Interplay of Structural and Optoelectronic Properties in Formamidinium Mixed Tin-Lead Triiodide Perovskites. Adv. Funct. Mater. 2018, 28, 1802803.

(19) Ross, R. T.; Nozik, A. J. Efficiency of Hot-Carrier Solar Energy Converters. J. Appl. Phys. 1982, 53, 3813-3818.

(20) Richter, J. M.; Branchi, F.; Valduga de Almeida Camargo, F.; Zhao, B.; Friend, R. H.; Cerullo, G.; Deschler, F. Ultrafast Carrier Thermalization in Lead Iodide Perovskite Probed with TwoDimensional Electronic Spectroscopy. Nat. Commun. 2017, 8, 376.

(21) Hsu, H.-Y.; Wang, C.-Y.; Fathi, A.; Shiu, J.-W.; Chung, C.-C.; Shen, P.-S.; Guo, T.-F.; Chen, P.; Lee, Y.-P.; Diau, E. W.-G. Femtosecond Excitonic Relaxation Dynamics of Perovskite on Mesoporous Films of $\mathrm{Al}_{2} \mathrm{O}_{3}$ and $\mathrm{NiO}$ Nanoparticles. Angew. Chem. 2014, 126, 9493-9496.

(22) Price, M. B.; Butkus, J.; Jellicoe, T. C.; Sadhanala, A.; Briane, A.; Halpert, J. E.; Broch, K.; Hodgkiss, J. M.; Friend, R. H.; Deschler, F. Hot-Carrier Cooling and Photoinduced Refractive Index Changes in Organic-Inorganic Lead Halide Perovskites. Nat. Commun. 2015, 6, 8420.

(23) Chen, K.; Barker, A. J.; Morgan, F. L. C.; Halpert, J. E.; Hodgkiss, J. M. Effect of Carrier Thermalization Dynamics on Light Emission and Amplification in Organometal Halide Perovskites. J. Phys. Chem. Lett. 2015, 6, 153-158.

(24) Shen, Q.; Ripolles, T. S.; Even, J.; Ogomi, Y.; Nishinaka, K.; Izuishi, T.; Nakazawa, N.; Zhang, Y.; Ding, C.; Liu, F.; et al. Slow Hot Carrier Cooling in Cesium Lead Iodide Perovskites. Appl. Phys. Lett. 2017, 111, 153903.

(25) Zhu, H.; Miyata, K.; Fu, Y.; Wang, J.; Joshi, P. P.; Niesner, D.; Williams, K. W.; Jin, S.; Zhu, X.-Y. Screening in Crystalline Liquids Protects Energetic Carriers in Hybrid Perovskites. Science 2016, 353, 1409-1413.

(26) Yang, J.; Wen, X.; Xia, H.; Sheng, R.; Ma, Q.; Kim, J.; Tapping, P.; Harada, T.; Kee, T. T. W.; Huang, F.; et al. Acoustic-Optical Phonon Up-Conversion and Hot-Phonon Bottleneck in Lead-Halide Perovskites. Nat. Commun. 2017, 8, 14120.

(27) Fang, H.-H.; Adjokatse, S.; Shao, S.; Even, J.; Loi, M. A. LongLived Hot-Carrier Light Emission and Large Blue Shift in Formamidinium Tin Triiodide Perovskites. Nat. Commun. 2018, 9, 243.

(28) Papagiorgis, P.; Protesescu, L.; Kovalenko, M. V.; Othonos, A.; Itskos, G. Long-Lived Hot Carriers in Formamidinium Lead Iodide Nanocrystals. J. Phys. Chem. C 2017, 121, 12434-12440.

(29) Jia, X.; Jiang, J.; Zhang, Y.; Qiu, J.; Wang, S.; Chen, Z.; Yuan, N.; Ding, J. Observation of Enhanced Hot Phonon Bottleneck Effect in 2D Perovskites. Appl. Phys. Lett. 2018, 112, 143903.

(30) Yang, Y.; Ostrowski, D. P.; France, R. M.; Zhu, K.; van de Lagemaat, J.; Luther, J. M.; Beard, M. C. Observation of a HotPhonon Bottleneck in Lead-Iodide Perovskites. Nat. Photonics 2016, $10,53-59$.

(31) Shen, Q.; Ripolles, T. S.; Even, J.; Zhang, Y.; Ding, C.; Liu, F.; Izuishi, T.; Nakazawa, N.; Toyoda, T.; Ogomi, Y.; et al. Ultrafast Selective Extraction of Hot Holes from Cesium Lead Iodide Perovskite Films. J. Energy Chem. 2018, 27, 1170-1174.

(32) Nah, S.; Spokoyny, B. M.; Soe, C. M. M.; Stoumpos, C. C.; Kanatzidis, M. G.; Harel, E. Ultrafast Imaging of Carrier Cooling in 
Metal Halide Perovskite Thin Films. Nano Lett. 2018, 18, 10441048.

(33) Li, M.; Bhaumik, S.; Goh, T. W.; Kumar, M. S.; Yantara, N.; Grätzel, M.; Mhaisalkar, S.; Mathews, N.; Sum, T. C. Slow Cooling and Highly Efficient Extraction of Hot Carriers in Colloidal Perovskite Nanocrystals. Nat. Commun. 2017, 8, 14350.

(34) Fu, J.; Xu, Q.; Han, G.; Wu, B.; Huan, C. H. A.; Leek, M. L.; Sum, T. C. Hot Carrier Cooling Mechanisms in Halide Perovskites. Nat. Commun. 2017, 8, 1300.

(35) Chen, J.; Messing, M. E.; Zheng, K.; Pullerits, T. CationDependent Hot Carrier Cooling in Halide Perovskite Nanocrystals. J. Am. Chem. Soc. 2019, 141, 3532-3540.

(36) Chang, M. H.; Hoffmann, M.; Anderson, H. L.; Herz, L. M. Dynamics of Excited-State Conformational Relaxation and Electronic Delocalization in Conjugated Porphyrin Oligomers. J. Am. Chem. Soc. 2008, 130, 10171-10178.

(37) Gong, J. Q.; Parkinson, P.; Kondratuk, D. V.; Gil-Ramírez, G.; Anderson, H. L.; Herz, L. M. Structure-Directed Exciton Dynamics in Templated Molecular Nanorings. J. Phys. Chem. C 2015, 119, 64146420.

(38) Shah, J.; Leite, R. C. C. Radiative Recombination from Photoexcited Hot Carriers in GaAs. Phys. Rev. Lett. 1969, 22, 13041307.

(39) Motisuke, P.; Argüello, C. A.; Leite, R. C. C. Hot Electron and Hot Phonon Contributions to Radiative Emission Spectra in CdS at High Excitation Intensities. Solid State Commun. 1975, 16, 763-765.

(40) Shah, J.; Lin, C.; Leheny, R. F.; DiGiovanni, A. E. Pump Wavelength Dependence of Hot Electron Temperature in GaAs. Solid State Commun. 1976, 18, 487-489.

(41) Leo, K.; Rühle, W. W. Influence of Carrier Lifetime on the Cooling of a Hot Electron-Hole Plasma in GaAs. Solid State Commun. 1987, 62, 659-662.

(42) Ryan, J. F.; Taylor, R. A.; Turberfield, A. J.; Maciel, A.; Worlock, J. M.; Gossard, A. C.; Wiegmann, W. Time-Resolved Photoluminescence of Two-Dimensional Hot Carriers in GaAsAlGaAs Heterostructures. Phys. Rev. Lett. 1984, 53, 1841-1844.

(43) Xu, Z. Y.; Tang, C. L. Picosecond Relaxation of Hot Carriers in Highly Photoexcited Bulk GaAs and GaAs-AlGaAs Multiple Quantum Wells. Appl. Phys. Lett. 1984, 44, 692-694.

(44) Ryan, J. F. Time-Resolved Photoluminescence for Quantum Well Semiconductor Heterostructures. Physica B+C 1985, 134, 403411.

(45) Shum, K.; Ho, P.; Alfano, R.; Welch, D.; Wicks, G.; Eastman, L. Dependence of Electron Temperature on Well Width in the $\mathrm{A}_{0.48} \mathrm{In}_{0.52} \mathrm{As} / \mathrm{Ga}_{0.47} \mathrm{In}_{0.53} \mathrm{As}$ Single-Quantum Well. IEEE J. Quantum Electron. 1986, 22, 1811-1815.

(46) Leo, K.; Rühle, W. W.; Ploog, K. Hot-Carrier Energy-Loss Rates in GaAs $/ \mathrm{Al}_{x} \mathrm{Ga}_{1-x}$ As Quantum Wells. Phys. Rev. B: Condens. Matter Mater. Phys. 1988, 38, 1947-1957.

(47) Leo, K.; Rühle, W. W.; Queisser, H. J.; Ploog, K. Reduced Dimensionality of Hot-Carrier Relaxation in GaAs Quantum Wells. Phys. Rev. B: Condens. Matter Mater. Phys. 1988, 37, 7121-7124.

(48) Leo, K.; Rühle, W. W.; Ploog, K. Hot Carrier Thermalization in GaAs/AlAs Superlattices. Solid-State Electron. 1989, 32, 1863-1867.

(49) Andersson, T. G.; Chen, Z.-G.; Xu, Z.-Y.; Xu, J.-Z.; Ge, W.-K. Hot Carrier Photoluminescence from Strained InxGa ${ }_{1 x}$ As/GaAs Single Quantum Wells. J. Cryst. Growth 1989, 95, 215-219.

(50) Nozik, A. J.; Parsons, C. A.; Dunlavy, D. J.; Keyes, B. M.; Ahrenkiel, R. K. Dependence of Hot Carrier Luminescence on Barrier Thickness in GaAs/AlGaAs Superlattices and Multiple Quantum Wells. Solid State Commun. 1990, 75, 297-301.

(51) Ge, W.; Xu, Z.; Li, Y.; Xu, Z.; Xu, J.; Zheng, B.; Zhuang, W. Hot Carrier Relaxation Processes and Nonequilibrium Phonon Effect in Multiple Quantum Well Structures. J. Lumin. 1990, 46, 137-145.

(52) Wehrenfennig, C.; Liu, M.; Snaith, H. J.; Johnston, M. B.; Herz, L. M. Homogeneous Emission Line Broadening in the Organo Lead Halide Perovskite $\mathrm{CH}_{3} \mathrm{NH}_{3} \mathrm{PbI}_{3-x} \mathrm{Cl}_{x}$. J. Phys. Chem. Lett. 2014, 5, $1300-1306$
(53) Dar, M. I.; Jacopin, G.; Meloni, S.; Mattoni, A.; Arora, N.; Boziki, A.; Zakeeruddin, S. M.; Rothlisberger, U.; Grätzel, M. Origin of Unusual Bandgap Shift and Dual Emission in Organic-Inorganic Lead Halide Perovskites. Sci. Adv. 2016, 2, No. e1601156.

(54) Wright, A. D.; Verdi, C.; Milot, R. L.; Eperon, G. E.; PérezOsorio, M. A.; Snaith, H. J.; Giustino, F.; Johnston, M. B.; Herz, L. M. Electron-Phonon Coupling in Hybrid Lead Halide Perovskites. Nat. Commun. 2016, 7, 11755.

(55) Arend, T. R.; Tönnies, M.; Reisbeck, P.; Rieckmann, C. J. P.; Kersting, R. Physical Vapor Deposition of Methylammonium Tin Iodide Thin Films. Phys. Status Solidi A 2017, 214, 1600796.

(56) Phuong, L. Q.; Braly, I. L.; Katahara, J. K.; Hillhouse, H. W.; Kanemitsu, Y. Nonlinear Photocarrier Recombination Dynamics in Mixed-Halide $\mathrm{CH}_{3} \mathrm{NH}_{3} \mathrm{~Pb}\left(\mathrm{I}_{1-x} \mathrm{Br}_{x}\right)_{3}$ Perovskite Thin Films. Appl. Phys. Express 2017, 10, 102401.

(57) Handa, T.; Aharen, T.; Wakamiya, A.; Kanemitsu, Y. Photoelectronic Properties of Lead-Free $\mathrm{CH}_{3} \mathrm{NH}_{3} \mathrm{SnI}_{3}$ Perovskite Solar Cell Materials and Devices. In Organic, Hybrid, and Perovskite Photovoltaics XIX; 2018; p 107371X.

(58) Milot, R. L.; Eperon, G. E.; Snaith, H. J.; Johnston, M. B.; Herz, L. M. Temperature-Dependent Charge-Carrier Dynamics in $\mathrm{CH}_{3} \mathrm{NH}_{3} \mathrm{PbI}_{3}$ Perovskite Thin Films. Adv. Funct. Mater. 2015, 25, 6218-6227.

(59) Straus, D. B.; Hurtado Parra, S.; Iotov, N.; Gebhardt, J.; Rappe, A. M.; Subotnik, J. E.; Kikkawa, J. M.; Kagan, C. R. Direct Observation of Electron-Phonon Coupling and Slow Vibrational Relaxation in Organic-Inorganic Hybrid Perovskites. J. Am. Chem. Soc. 2016, 138, 13798-13801.

(60) Yu, P. Y.; Cardona, M. Fundamentals of Semiconductors: Physics and Materials Properties, 4th ed.; Springer, 2010.

(61) Mooradian, A.; Fan, H. Y. Recombination Emission in InSb. Phys. Rev. 1966, 148, 873-885.

(62) Verma, S. D.; Gu, Q.; Sadhanala, A.; Venugopalan, V.; Rao, A. Slow Carrier Cooling in Hybrid $\mathrm{Pb}-\mathrm{Sn}$ Halide Perovskites. ACS Energy Lett. 2019, 4, 736-740.

(63) Stoumpos, C. C.; Malliakas, C. D.; Kanatzidis, M. G. Semiconducting Tin and Lead Iodide Perovskites with Organic cations: Phase Transitions, High Mobilities, and Near-Infrared Photoluminescent Properties. Inorg. Chem. 2013, 52, 9019-9038.

(64) Gupta, S.; Cahen, D.; Hodes, G. How $\mathrm{SnF}_{2}$ Impacts the Material Properties of Lead-Free Tin Perovskites. J. Phys. Chem. C 2018, 122, 13926-13936.

(65) Ma, J.; Wang, L.-W. Nanoscale Charge Localization Induced by Random Orientations of Organic Molecules in Hybrid Perovskite $\mathrm{CH}_{3} \mathrm{NH}_{3} \mathrm{PbI}_{3}$. Nano Lett. 2015, 15, 248-253.

(66) Juarez-Perez, E. J.; Sanchez, R. S.; Badia, L.; Garcia-Belmonte, G.; Kang, Y. S.; Mora-Sero, I.; Bisquert, J. Photoinduced Giant Dielectric Constant in Lead Halide Perovskite Solar Cells. J. Phys. Chem. Lett. 2014, 5, 2390-2394.

(67) Motta, C.; El-Mellouhi, F.; Kais, S.; Tabet, N.; Alharbi, F.; Sanvito, S. Revealing the Role of Organic Cations in Hybrid Halide Perovskite $\mathrm{CH}_{3} \mathrm{NH}_{3} \mathrm{PbI}_{3}$. Nat. Commun. 2015, 6, 7026 .

(68) March, S. A.; Riley, D. B.; Clegg, C.; Webber, D.; Liu, X.; Dobrowolska, M.; Furdyna, J. K.; Hill, I. G.; Hall, K. C. Four-Wave Mixing in Perovskite Photovoltaic Materials Reveals Long Dephasing Times and Weaker Many-Body Interactions than GaAs. ACS Photonics 2017, 4, 1515-1521.

(69) Frost, J. M.; Butler, K. T.; Brivio, F.; Hendon, C. H.; Van Schilfgaarde, M.; Walsh, A. Atomistic Origins of High-Performance in Hybrid Halide Perovskite Solar Cells. Nano Lett. 2014, 14, 25842590.

(70) He, H.; Yu, Q.; Li, H.; Li, J.; Si, J.; Jin, Y.; Wang, N.; Wang, J.; He, J.; Wang, X.; et al. Exciton Localization in Solution-Processed Organolead Trihalide Perovskites. Nat. Commun. 2016, 7, 10896.

(71) Wright, A. D.; Milot, R. L.; Eperon, G. E.; Snaith, H. J.; Johnston, M. B.; Herz, L. M. Band-Tail Recombination in Hybrid Lead Iodide Perovskite. Adv. Funct. Mater. 2017, 27, 1700860. 
(72) Manser, J. S.; Kamat, P. V. Band Filling with Free Charge Carriers in Organometal halide Perovskites. Nat. Photonics 2014, 8, 737-743.

(73) Yang, Y.; Yan, Y.; Yang, M.; Choi, S.; Zhu, K.; Luther, J. M.; Beard, M. C. Low Surface Recombination Velocity in Solution-Grown $\mathrm{CH}_{3} \mathrm{NH}_{3} \mathrm{PbBr}_{3}$ Perovskite Single Crystal. Nat. Commun. 2015, 6, 7961.

(74) Whalley, L. D.; Frost, J. M.; Morgan, B. J.; Walsh, A. Impact of Nonparabolic Electronic Band Structure on the Optical and Transport Properties of Photovoltaic Materials. Phys. Rev. B: Condens. Matter Mater. Phys. 2019, 99, 085207.

(75) Suhling, K.; French, P. M. W.; Phillips, D. Time-Resolved Fluorescence Microscopy. Photochem. Photobiol. Sci. 2005, 4, 13-22.

(76) Chan, F. T. S.; Kaminski, C. F.; Kaminski Schierle, G. S. HomoFRET Fluorescence Anisotropy Imaging as a Tool to Study Molecular Self-Assembly in Live Cells. ChemPhysChem 2011, 12, 500-509.

(77) Herz, L. M.; Phillips, R. T. Effects of Interchain Interactions, Polarization Anisotropy, and Photo-Oxidation on the Iltrafast Photoluminescence Decay from a Polyfluorene. Phys. Rev. B: Condens. Matter Mater. Phys. 2000, 61, 13691-13697.

(78) Zemskii, V. I.; Zakharchenya, B. P.; Mirlin, D. N. Polarization of Hot Photoluminescence in semiconductors of the GaAs Type. JETP Lett. 1976, 24, 82-85.

(79) Portella, M. T.; Bigot, J.-Y.; Schoenlein, R. W.; Cunningham, J. E.; Shank, C. V. k-Space Carrier Dynamics in GaAs. Appl. Phys. Lett. 1992, 60, 2123-2125.

(80) Rivett, J. P. H.; Tan, L. Z.; Price, M. B.; Bourelle, S. A.; Davis, N. J. L. K.; Xiao, J.; Zou, Y.; Middleton, R.; Sun, B.; Rappe, A. M.; et al. Long-Lived Polarization Memory in the Electronic States of Lead-Halide Perovskites from Local Structural Dynamics. Nat. Commun. 2018, 9, 3531.

(81) Yaffe, O.; Guo, Y.; Tan, L. Z.; Egger, D. A.; Hull, T.; Stoumpos, C. C.; Zheng, F.; Heinz, T. F.; Kronik, L.; Kanatzidis, M. G.; et al. Local Polar Fluctuations in Lead Halide Perovskite Crystals. Phys. Rev. Lett. 2017, 118, 136001.

(82) Wu, X.; Tan, L. Z.; Shen, X.; Hu, T.; Miyata, K.; Trinh, M. T.; Li, R.; Coffee, R.; Liu, S.; Egger, D. A.; et al. Light-Induced Picosecond Rotational Disordering of the Inorganic Sublattice in Hybrid Perovskites. Sci. Adv. 2017, 3, No. e1602388.

(83) Beecher, A. N.; Semonin, O. E.; Skelton, J. M.; Frost, J. M.; Terban, M. W.; Zhai, H.; Alatas, A.; Owen, J. S.; Walsh, A.; Billinge, S. J. L. Direct Observation of Dynamic Symmetry Breaking Above Room Temperature in Methylammonium Lead Iodide Perovskite. ACS Energy Lett. 2016, 1, 880-887.

(84) Etienne, T.; Mosconi, E.; De Angelis, F. Dynamical Origin of the Rashba Effect in Organohalide Lead Perovskites: A Key to Suppressed Carrier Recombination in Perovskite Solar Cells? J. Phys. Chem. Lett. 2016, 7, 1638-1645.

(85) Umari, P.; Mosconi, E.; De Angelis, F. Relativistic GW Calculations on $\mathrm{CH}_{3} \mathrm{NH}_{3} \mathrm{PbI}_{3}$ and $\mathrm{CH}_{3} \mathrm{NH}_{3} \mathrm{SnI}_{3}$ Perovskites for Solar Cell Applications. Sci. Rep. 2015, 4, 4467.

(86) Kubicki, D. J.; Prochowicz, D.; Hofstetter, A.; Péchy, P.; Zakeeruddin, S. M.; Grätzel, M.; Emsley, L. Cation Dynamics in Mixed-Cation $(\mathrm{MA})_{x}(\mathrm{FA})_{1-x} \mathrm{PbI}_{3}$ Hybrid Perovskites from SolidState NMR. J. Am. Chem. Soc. 2017, 139, 10055-10061.

(87) Motta, C.; El-Mellouhi, F.; Sanvito, S. Exploring the Cation Dynamics in Lead-Bromide Hybrid Perovskites. Phys. Rev. B: Condens. Matter Mater. Phys. 2016, 93, 235412.

(88) Fabini, D. H.; Siaw, T. A.; Stoumpos, C. C.; Laurita, G.; Olds, D.; Page, K.; Hu, J. G.; Kanatzidis, M. G.; Han, S.; Seshadri, R. Universal Dynamics of Molecular Reorientation in Hybrid Lead Iodide Perovskites. J. Am. Chem. Soc. 2017, 139, 16875-16884.

(89) Zhai, Y.; Sheng, C. X.; Zhang, C.; Vardeny, Z. V. Ultrafast Spectroscopy of Photoexcitations in Organometal Trihalide Perovskites. Adv. Funct. Mater. 2016, 26, 1617-1627.

(90) Wang, K.-H.; Li, L.-C.; Shellaiah, M.; Sun, K. W. Structural and Photophysical Properties of Methylammonium Lead Tribromide $\left(\mathrm{MAPbBr}_{3}\right)$ Single Crystals. Sci. Rep. 2017, 7, 13643.
(91) Noh, J. H.; Im, S. H.; Heo, J. H.; Mandal, T. N.; Seok, S. I. Chemical Management for Colorful, Efficient, and Stable InorganicOrganic Hybrid Nanostructured Solar Cells. Nano Lett. 2013, 13, 1764-1769.

(92) Schueller, E. C.; Laurita, G.; Fabini, D. H.; Stoumpos, C. C.; Kanatzidis, M. G.; Seshadri, R. Crystal Structure Evolution and Notable Thermal Expansion in Hybrid Perovskites Formamidinium Tin Iodide and Formamidinium Lead Bromide. Inorg. Chem. 2018, 57, 695-701.

(93) Mitzi, D. B.; Liang, K. Synthesis, Resistivity, and Thermal Properties of the Cubic Perovskite $\mathrm{NH}_{2} \mathrm{CH}=\mathrm{NH}_{2} \mathrm{SnI}_{3}$ and Related Systems. J. Solid State Chem. 1997, 134, 376-381.

(94) Lee, S. J.; Shin, S. S.; Kim, Y. C.; Kim, D.; Ahn, T. K.; Noh, J. H.; Seo, J.; Seok, S. I. Fabrication of Efficient Formamidinium Tin Iodide Perovskite Solar Cells through $\mathrm{SnF}_{2}-$ Pyrazine Complex. J. Am. Chem. Soc. 2016, 138, 3974-3977.

(95) Koh, T. M.; Krishnamoorthy, T.; Yantara, N.; Shi, C.; Leong, W. L.; Boix, P. P.; Grimsdale, A. C.; Mhaisalkar, S. G.; Mathews, N. Formamidinium Tin-Based Perovskite with Low $\mathrm{E}_{g}$ for Photovoltaic Applications. J. Mater. Chem. A 2015, 3, 14996-15000. 